\title{
Standardized in vitro analysis of the degradability of hyaluronic acid fillers by hyaluronidase
}

Bettina Alexandra Buhren ${ }^{1}$, Holger Schrumpf ${ }^{1}$, Edwin Bölke², Kai Kammers ${ }^{3}$ and Peter Arne Gerber ${ }^{\text {** }}$

\begin{abstract}
Background: Hyaluronidase is a hyaluronic acid (HA) metabolizing enzyme, which is approved as an adjuvant for infiltration anesthesia. The "off-label" use of hyaluronidase is regarded as gold standard for the management of HAfiller-associated complications. Yet, up to date there are only few studies that have systematically assessed the degradability of different HA-fillers by hyaluronidase.

Objective: To analyze the interactions of HA-fillers and hyaluronidase in a time-dependent manner using a novel standardized in vitro approach.

Methods: Comparable HA-fillers, Belotero Balance Lidocaine (BEL; Merz), Emervel classic (EMV; Galderma) and Juvederm Ultra 3 (JUV; Allergan), were incubated with a fluorescent dye and bovine hyaluronidase (HYAL; Hylase "Dessau", Riemser) or control ( $\mathrm{NaCl}$ ) and monitored by time-lapse videomicroscopy. The degradation of HA-fillers was assessed as decrease in fluorescence intensity of HA-filler plus hyaluronidase vs. HA-filler plus control, quantified by computerassisted image analysis (ImageJ).

Results: Hyaluronidase showed a significant degradation of the HA-fillers BEL and EMV. Degradation was measurable at $5 \mathrm{~h}$ (BEL) and $7 \mathrm{~h}(\mathrm{EMV})$, respectively; significance was reached at $14 \mathrm{~h}$ (BEL) and $13 \mathrm{~h}$ (EMV). No effect of hyaluronidase was observed for JUV.

Conclusion: Time-lapse microscopy enables systematically, standardized, comparative in vitro analyses of the interactions of hyaluronidase and HA-fillers.
\end{abstract}

\section{Background}

Hyaluronic acid (hyaluronan, HA) is a non-sulfated glycosaminoglycan (GAG) and an essential part of the skin's extracellular matrix (ECM) $[1,2]$. A decrease in the skin's HA content is considered as main characteristic of skin aging $[3,4]$. Today, the injection of reversible HA-based dermal fillers is regarded as gold standard for tissue augmentation, deep skin hydration or facial recontouring $[5,6]$. Potential complications of filler treatments range from unaesthetic overcorrections, Tyndall effect or lower eyelid edema following tear-trough augmentation,

\footnotetext{
*Correspondence: peterarne.gerber@med.uni-duesseldorf.de

${ }^{1}$ Departments of Dermatology, Medical Faculty, Research Laboratory for Dermatology and Immunology, Heinrich-Heine-University

Duesseldorf, University Hospital Duesseldorf, Duesseldorf, Germany

Full list of author information is available at the end of the article
}

to granulomas, infections, up to tissue necrosis or even blindness due to vascular occlusions [1]. The availability of a specific antidote, hyaluronidase, for the management of complications of filler treatments is one major reason for the preferred use of HA-based fillers over other injectable fillers, such as calcium hydroxylapatite (CHA) or poly-L-lactic acid [1, 7-9]. Since the timely infiltration of hyaluronidase may degrade HA-fillers and may rescue from more severe vascular complications, the immediate availability of hyaluronidase is regarded a necessity for every physician that injects HA $[8,10,11]$. Despite the availability of hyaluronidase, it is controversially discussed whether all HA-fillers can be degraded by hyaluronidase assimilably effective. A difference in or even resistance to "degradability" may be attributed to the concentration of HA in the filler, the degree of cross-linking, 
and/or its cohesive properties [12,13]. Taking in account the potential risks associated with filler injections, the proven degradability of an HA-filler by hyaluronidase can be regarded as "safety-feature" and potential competitive edge over other manufacturers of dermal fillers.

Against this background, we here set out to systematically analyze the degradability of three commonly used HA-fillers by bovine hyaluronidase in a time dependent manner, using a novel standardized video-microscopic in vitro approach.

\section{Methods}

For our analysis we used three comparable commercially available HA-fillers, Belotero Balance Lidocain (BEL; monophasic double cross-linked, BDDE, HA $22.5 \mathrm{mg} /$ $\mathrm{ml}$, with lidocaine; Merz Pharmaceuticals GmbH, Frankfurt, Germany; manufactured by ANTEIS SA, Geneva, Switzerland), Emervel classic (EMV; cross-linked, biphasic, sizing, BDDE, HA $20.0 \mathrm{mg} / \mathrm{ml}$, lidocaine; Galderma; manufactured by Q-Med AB, Uppsala, Sweden), and Juvederm Ultra 3 (JUV; cross-linked "Hyalcross", BDDE, HA $24.0 \mathrm{mg} / \mathrm{ml}$, lidocaine; Pharm-Allergan, Irvine, CA, USA) [14]. First, $50 \mu \mathrm{l}$ of each filler were mixed with $10 \mu \mathrm{l}$ of a green fluorescent cell linker dye (PKH67, Sigma) in addition to 10 units $(\mathrm{U} / \mathrm{ml})$ of bovine testicular hyaluronidase (HYAL; Hylase "Dessau", Riemser Pharma, Greifswald, Germany), which represents the standard hyaluronidase used in Germany, or an equal volume of $\mathrm{NaCl}$ and placed in 24-well plates (BD Bioscience). Next, the now fluorescent gel was placed in a larger volume of $350 \mu \mathrm{l}$ of serum-free keratinocyte medium (SFM, Thermo Fisher Scientific) and wells were placed in a time-lapse videomicroscopy workstation (Zeiss Axiovert 200 M, Carl Zeiss Microscopy GmbH, Göttingen, Germany; $\left.37{ }^{\circ} \mathrm{C}, 5 \% \mathrm{CO}_{2}\right)$. Depending on the integrity of the HAfiller the fluorescent dye remained either inclosed in the gel, resulting in high fluorescence intensity, or the dye was released and diluted in the surrounding medium, resulting in a decrease in fluorescence intensity. Fluorescence intensity was recorded over $20 \mathrm{~h}$ (5× magnification, FITC channel). Images were acquired using a high resolution monochromatic CCD chip (Zeiss). The workstation was driven by control software Axiovision 4.7 (Zeiss). The degradation of the HA-fillers was assessed as difference in fluorescence of HA-filler plus HYAL vs. HAfiller plus control (CTR; $\mathrm{NaCl}$ ), $n \leq 6$ per condition, quantified by computer-assisted image analysis (BioVoxxel Fiji Image $1.49 \mathrm{~m}$ ). Figure 1 shows a schematic illustration of our experimental setup.

Raw fluorescence intensities were measured every $10 \mathrm{~min}$ and six consecutive measurements were averaged to obtain a robust value for each full hour. For easier visualization and interpretation, results were displayed on a multiplicative scale, i.e., averaged fluorescence intensities for each full hour were divided by the corresponding fluorescence intensities at time point $0 \mathrm{~h}$. A value of, e.g., 0.5 in Fig. 2a-c illustrates that 50\% fluorescence intensity is remaining compared to $t=0$. At each time point, normalized fluorescence intensities between two groups were compared using a nonparametric Wilcoxon ranksum test. Differences between two conditions with calculated $p$ values smaller than 0.05 were declared statistically significant. In Fig. $2 \mathrm{w}$ all conditions were summarized by normalizing HYAL-treated conditions against their respective CRT-values (zero line).

\section{Results}

All conditions (BEL + HYAL, BEL + CTR, EMV + HYAL, EMV + CTR, JUV + HYAL, JUV + CTR) showed comparable fluorescence intensities at $0 \mathrm{~h}$ (Fig. $2 \mathrm{~d}-\mathrm{i}$ ). Moreover, for all conditions we documented a mild to moderate fluctuation of the overall over the course of the experiment. Significant separations of the curves for HYAL- vs. CTR-treated fillers and hence a degradation of the fillers were observed only for BEL and EMV (Fig. 2v). A degradation of BEL by HYAL $(n=5)$ was observed at $5 \mathrm{~h}$ and reached significance $(p=0.03)$ starting at $14 \mathrm{~h}$ (Fig. 2b). A degradation of EMV by HYAL $(n=6)$ was observed at $7 \mathrm{~h}$ and reached significance $(p=0.04)$ starting at $13 \mathrm{~h}$ (Fig. 2c). The strongest overall degradation was observed for BEL (Fig. 2b, s, v). No significant separation of degradation-curves and hence no effect of HYAL was observed for JUV $(n=4)$ (Fig. 2a, e, k,q).

\section{Discussion}

Few studies have systematically assessed the degradability of HA-fillers by hyaluronidase [12-16]. In 2007 Sall et al. used a test based on the colorimetric determination of $\mathrm{N}$-acetyl-D-glucosamine released from 11 different HA-fillers by bovine hyaluronidase [16]. Three years later Jones et al. applied an in vitro approach by analysis of the degradation products of 3 different HA-fillers treated with ovine hyaluronidase using sizeexclusion chromatography [12]. In line with the results of our analysis, both studies reported the strongest resistance to degradation for the highly cross-linked $24 \mathrm{mg} / \mathrm{ml} \mathrm{HA}$-filler (JUV) [12, 16]. The biphasic $20 \mathrm{mg} /$ ml HA-filler Restylane (equal product as Emervel classic, EMV) was reported as most sensitive [16]. In 2014 Rao et al. used a photographic approach to visually compare the interaction of human recombinant hyaluronidase (Hylenex, Halozyme Therapeutics, San Diego, CA, USA) with 4 different HA-fillers [13]. Again, the authors reported that Restylane (EMV) was degraded by hyaluronidase most effectively in a dose-dependent manner. Contrary to our results Belotero (BEL) 


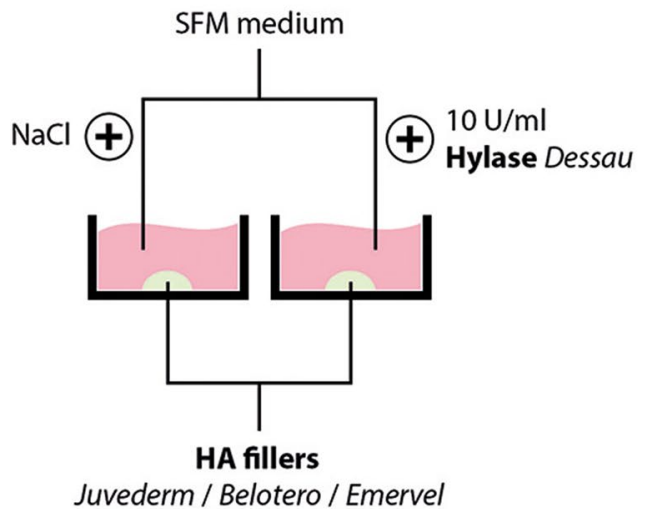

(†)

Green fluorescence dye PKH67

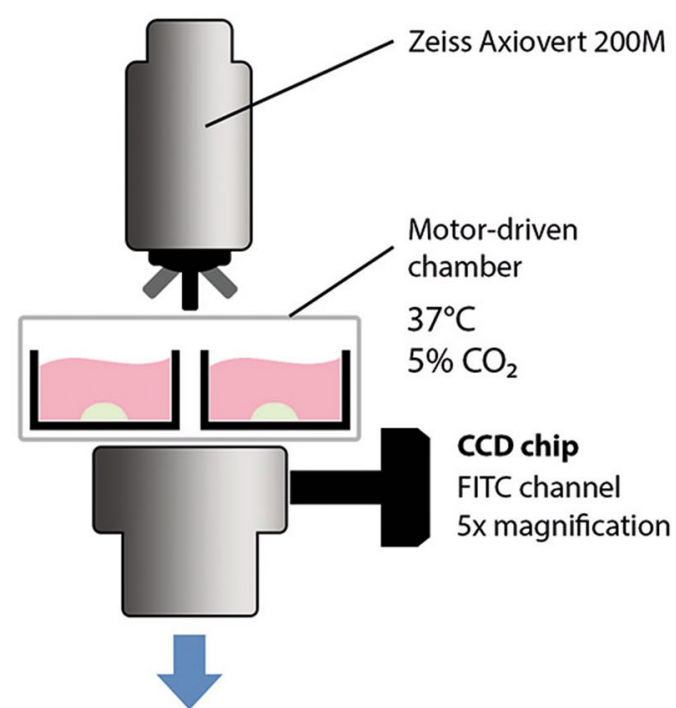

Time-lapse recording of fluorescence intensity/ degradation of HA fillers over 20 hrs

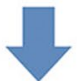

Analysis

BioVoxxel Fiji ImageJ

Fig. 1 Schematic illustration of the experimental setup

retained its form most followed by Juvederm (JUV). Most recently, Juhász et al. conducted an in vivo human study [14]. Herein, 7 different HA-fillers, including Belotero (BEL), Restylane (EMV), and Juvederm (JUV), were injected into the back skin of 15 participants, followed by secondary injections of ovine hyaluronidase (20 or 40 units; Vitrase, Valeant Pharmaceuticals, Laval, Canada) or saline. Degradation of HA-fillers was monitored by palpation over the following 14 days. In summary, over the entire period of the observation all HA-fillers treated with hyaluronidase (20 and $40 \mathrm{U}$ ) showed a significant decrease in volume. In line with our analysis, Belotero (BEL) was found to be the fastest to dissolve.
Taken together, cited studies show a significant heterogeneity with regard to analyzed HA-fillers, hyaluronidases, and most notably experimental setups and techniques of analysis. As opposed to all other setups our approach is characterized by a high level of standardization, researcher-independent, computer-assisted quantification, as well as the opportunity to follow the interactions of HA-fillers and hyaluronidase over a complete time course due to time-lapse video documentation. Of note, we observed a mild to moderate fluctuation of the baseline- or CTR-fluorescence intensities for each different filler over the course of the experiment. This fluctuation is likely caused by a change in the shape of the filler, which is also evident from the representative

(See figure on next page.)

Fig. 2 Effect of hyaluronidase on three different HA-fillers in vitro. Time-lapse videomicroscopy fluorescence analysis of the HA-fillers a Juvederm Ultra 3 (JUV), b Belotero Balance Lidocain (BEL) and c Emervel classic (EMV) incubated with a fluorescent dye and bovine hyaluronidase (HYAL; blue dots) or control ( $\mathrm{NaCl}$; black circles) in hourly intervals over $20 \mathrm{~h}$. $\mathbf{d}$-u Representative fluorescence images obtained at $0 \mathrm{~h}(\mathbf{d}-\mathbf{i}), 10 \mathrm{~h} \mathrm{(j-0)}$, and $20 \mathrm{~h}$ (p-u) for JUV (d, j, p with NaCl; e, k, q with HYAL), BEL (f, I, $\mathbf{r}$ with NaCl; $\mathbf{g}, \mathbf{m}, \mathbf{s}$ with HYAL), and EMV (h, $\mathbf{n}, \mathbf{t}$ with NaCl; $\mathbf{j}, \mathbf{o}$, u with HYAL). Red asterisks mark time points showing statistically significant differences of fluorescence intensity between two groups $\left({ }^{*} p<0.05,{ }^{*} p<0.01\right)$. v Summary of fluorescence intensities for JUV (blue dots), BEL (red dots), EMV (green dots) normalized against their respective NaCL-CTRs (zero line) 


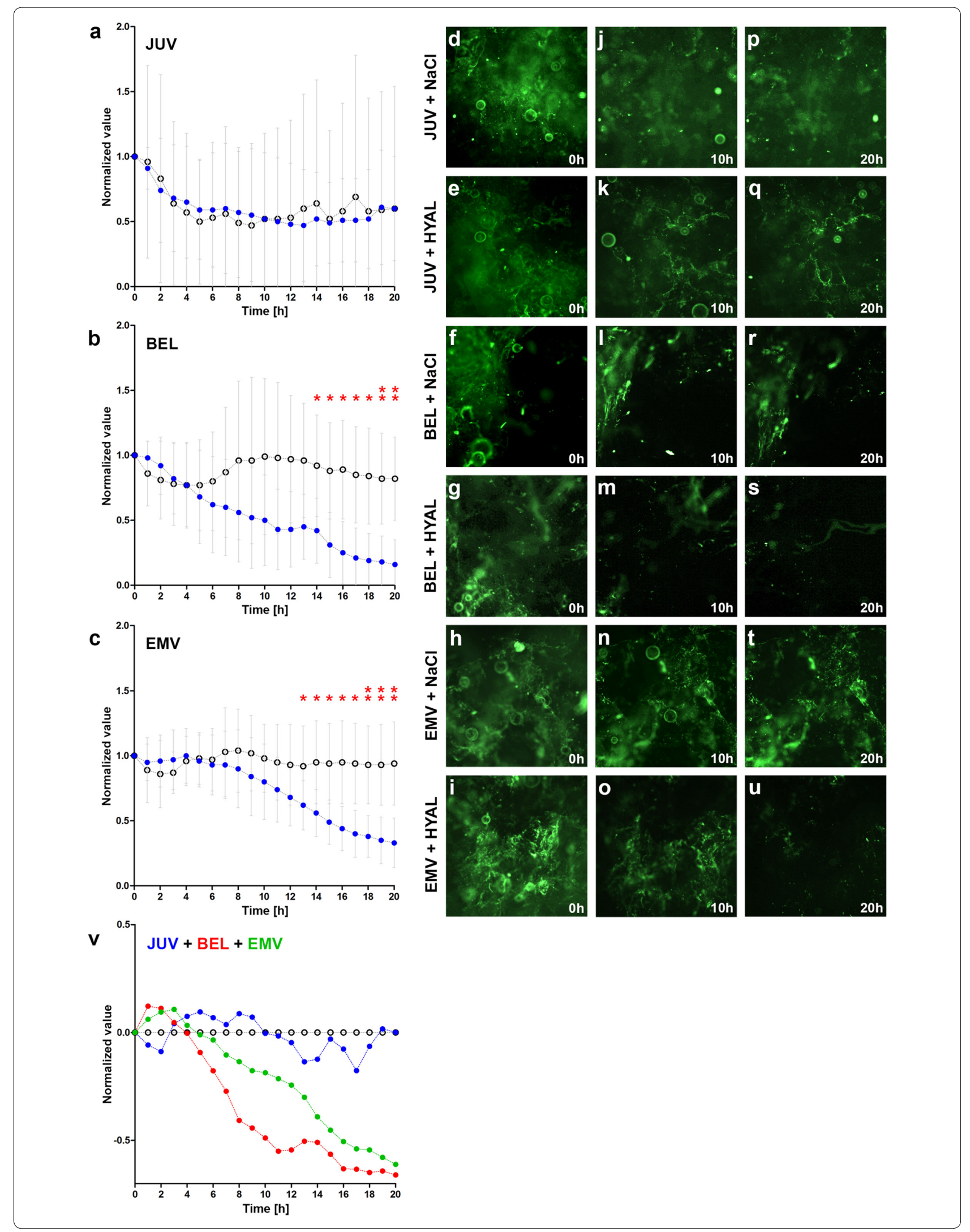


images displayed in Fig. 2 (e.g., d, j, p; f, l, r; h, n, t). Yet, only an effective degradation of the filler and hence dilution of the dye into the medium will result in significant separations of the curves for HYAL- vs. CTR-treated fillers, which were observed for BEL and EMV. To clarify these results we plotted all values for HYAL-treated fillers against their NACL-treated controls (CTR) as displayed in Fig. 2v. In conclusion, our results are in line with previous studies, showing that hyaluronidase effectively degrades BEL and EMV $[13,14,16]$.

Our results are furthermore confirmed by studies that assessed other aspects of the interaction of hyaluronidase and respective HA-fillers. In $2011 \mathrm{Kim}$ et al. used a rabbit ear model to demonstrate that hyaluronidase effectively prevents skin necrosis if it is injected within $4 \mathrm{~h}$ after vascular occlusion of an artery using Restylane (EMV) [10]. Recently, Wang et al. used the same rabbit ear model to show that a subcutaneous injection of hyaluronidase is more effective than the intra-arterial injection. Moreover, the authors could prove that hyaluronidase effectively degrades EMV within $1 \mathrm{~h}$ [17]. Finally, Menzinger et al. reported that hyaluronidase effectively and dose-dependently degraded EMV in a murine model in vivo [18].

With regard to the interaction of hyaluronidase and JUV results are more inconsistent. Whereas Sall et al. and Jones et al. reported the strongest resistance to degradation against bovine or ovine hyaluronidase $[12,16]$, Rao et al. as well as Juhász et al. demonstrated that ovine or recombinant human hyaluronidase effectively degrades JUV $[13,14]$. These controversial results could be related to differences in applied hyaluronidases (bovine, ovine, recombinant human) and respective doses, durations of incubation or experimental setups. Proposed hypotheses state that higher contents of HA as well as cross-linking-techniques have a strong effect on resistance against hyaluronidase. The strong degree of crosslinking of monophasic JUV may limit the access by the enzyme to its HA substrate, whereas the biphasic nature of EMV and its distinct particles offer a greater surface to attack [16]. In our analysis we also found that the filler with the highest content of HA (JUV, $24 \mathrm{mg} / \mathrm{ml}$ ) was most resistant to degradation as compared to fillers with lower concentrations (BEL, $22.5 \mathrm{mg} / \mathrm{ml}$; EMV, $20 \mathrm{mg} / \mathrm{ml}$ ). However, we found that the monophasic BEL was comparably sensitive to degradation as the biphasic EMV.

Taken together, our study demonstrates that timelapse videomicroscopy represents an elegant technique to assess the degradability of HA-fillers by hyaluronidase in a time-dependent manner. In this pilot-study we did not assess the effect of different doses or types of hyaluronidase. Likely higher doses of hyaluronidase may suffice to also degrade JUV, as it is evident from previous studies [13, 14]. Also higher doses of hyaluronidase will likely result in a faster degradation of HA-fillers within the first hour, like it is often observed in the in vivo or clinical situation [17]. Taking in account that hyaluronidase will not only degrade the HA-filler but also HA in the surrounding ECM it is a reasonable concern that the injection of high doses of hyaluronidase may result in a deficit of physiological HA in the treated area. Yet, the half-life and turn-over of non-stabilized, physiological HA in skin is only about $24 \mathrm{~h}$, implying that equilibrium is always established within a few hours $[19,20]$. In line with this hypothesis, to the best of our knowledge there are no reports on tissue deficits even after application of excessive doses of hyaluronidase, e.g., in cases of vascular occlusions following HA-filler injections. With regard to the molecular mechanisms, our results suggest that the content of $\mathrm{HA}$ and technique of cross-linking, but not the mono- or biphasic nature of a filler are the main factors that determine sensitivity to hyaluronidase. Future studies could extend our approach to dose-response analyses and a broader range of different HA-fillers and hyaluronidases.

\section{Authors' contributions}

$\mathrm{BB}, \mathrm{HS}$ and PAG carried out the studies, participated in collecting data, and drafted the manuscript. KK performed the statistical analysis and participated in its design. EB helped to draft the manuscript. All authors read and approved the final manuscript.

\section{Author details \\ ${ }^{1}$ Departments of Dermatology, Medical Faculty, Research Laboratory for Dermatology and Immunology, Heinrich-Heine-University Duesseldorf, University Hospital Duesseldorf, Duesseldorf, Germany. ${ }^{2}$ Departments of Radiation Oncology, Medical Faculty, Research Laboratory for Dermatology and Immunology, Heinrich-Heine-University Duesseldorf, University Hospital Duesseldorf, Duesseldorf, Germany. ${ }^{3}$ Division of Biostatistics and Bioinformat- ics, Department of Oncology, Sidney Kimmel Comprehensive Cancer Center, Johns Hopkins University School of Medicine, Baltimore, MD, USA.}

\section{Competing interests}

PAG has received honoraria for presentations from Galderma and Merz. PAG has received honoraira for advisory consultations from Galderma. PAG has received research funding from Riemser Pharma and Galderma. The other authors declare that they have no competing interests.

Availability of data and materials

All data and materials can be found in "Methods" section or can be accessed via Dr. Buhren and Prof. Dr. Gerber.

Consent for publication

Every author has given consent for publication.

Ethics approval and consent to participate

An ethical proposal was not necessary because no human beings/animals or their tissue were involved.

\section{Funding}

Not applicable.

\section{Publisher's Note}

Springer Nature remains neutral with regard to jurisdictional claims in published maps and institutional affiliations. 
Received: 23 April 2018 Accepted: 31 July 2018

Published online: 20 August 2018

\section{References}

1. Buhren BA, Schrumpf H, Hoff NP, Bolke E, Hilton S, Gerber PA. Hyaluronidase: from clinical applications to molecular and cellular mechanisms. Eur J Med Res. 2016;21:5.

2. Juhlin L. Hyaluronan in skin. J Intern Med. 1997;242:61-6.

3. Papakonstantinou E, Roth M, Karakiulakis G. Hyaluronic acid: a key molecule in skin aging. Dermatoendocrinol. 2012;4:253-8.

4. Meyer $L$, Stern R. Age-dependent changes of hyaluronan in human skin. J Invest Dermatol. 1994;102:385-9.

5. Narins RS, Brandt FS, Dayan SH, Hornfeldt CS. Persistence of nasolabial fold correction with a hyaluronic acid dermal filler with retreatment: results of an 18-month extension study. Dermatol Surg. 2011;37:644-50.

6. Narins RS, Coleman WP 3rd, Donofrio LM, et al. Improvement in nasolabial folds with a hyaluronic acid filler using a cohesive polydensified matrix technology: results from an 18-month open-label extension trial. Dermatol Surg. 2010;36(Suppl 3):1800-8.

7. Hilton S, Schrumpf H, Buhren BA, Bolke E, Gerber PA. Hyaluronidase injection for the treatment of eyelid edema: a retrospective analysis of 20 patients. Eur J Med Res. 2014:19:30.

8. Hirsch RJ, Brody HJ, Carruthers JD. Hyaluronidase in the office: a necessity for every dermasurgeon that injects hyaluronic acid. J Cosmet Laser Ther. 2007;9:182-5.

9. Hirsch RJ, Cohen JL, Carruthers JD. Successful management of an unusual presentation of impending necrosis following a hyaluronic acid injection embolus and a proposed algorithm for management with hyaluronidase. Dermatol Surg. 2007:33:357-60.

10. Kim DW, Yoon ES, Ji YH, Park SH, Lee BI, Dhong ES. Vascular complications of hyaluronic acid fillers and the role of hyaluronidase in management. $J$ Plast Reconstr Aesthet Surg. 2011;64:1590-5.
11. Jahn K, Homey B. Gerber PA [Management of complications after aesthetic hyaluronic acid injections]. Hautarzt. 2014;65:851-3.

12. Jones $D$, Tezel $A$, Borrell $M$. In vitro resistance to degradation of hyaluronic acid dermal fillers by ovine testicular hyaluronidase. Dermatol Surg. 2010;36:804-9.

13. Rao V, Chi S, Woodward J. Reversing facial fillers: interactions between hyaluronidase and commercially available hyaluronic-acid based fillers. J Drugs Dermatol. 2014;13:1053-6.

14. Juhasz MLW, Levin MK, Marmur ES. The kinetics of reversible hyaluronic acid filler injection treated with hyaluronidase. Dermatol Surg. 2017:43:841-7.

15. Hwang E, Song YS. Quantitative correlation between hyaluronic acid filler and hyaluronidase. J Craniofac Surg. 2017;28:838-41.

16. Sall I, Ferard G. Comparison of the sensitivity of 11 crosslinked hyaluronic acid gels to bovine testis hyaluronidase. Polym Degrad Stabil. 2007:92:915-9.

17. Wang M, Li W, Zhang Y, Tian W, Wang H. Comparison of intra-arterial and subcutaneous testicular hyaluronidase injection treatments and the vascular complications of hyaluronic acid filler. Dermatol Surg. 2017:43:246-54.

18. Menzinger S, Kaya A, Saurat JH, Kaya G. Injected hyaluronidase reduces the volume of exogenous hyaluronate fillers in mice and results in clinical improvement in a patient with pretibial myxedema. Dermatopathology (Basel). 2016;3:61-7.

19. Bohaumilitzky L, Huber AK, Stork EM, Wengert S, Woelf F, Boehm H. A trickster in disguise: Hyaluronan's ambivalent roles in the matrix. Front Oncol. 2017;7:242.

20. Buhren BA, Schrumpf H, Hoff NP, Bölke E, Hilton S, Gerber PA. Hyaluronidase: from clinical applications to molecular and cellular mechanisms. Eur J Med Res. 2016;13(21):5. https://doi.org/10.1186/s40001-016-0201-5. Review.
Ready to submit your research? Choose BMC and benefit from:

- fast, convenient online submission

- thorough peer review by experienced researchers in your field

- rapid publication on acceptance

- support for research data, including large and complex data types

- gold Open Access which fosters wider collaboration and increased citations

- maximum visibility for your research: over $100 \mathrm{M}$ website views per year

At BMC, research is always in progress.

Learn more biomedcentral.com/submissions 